\title{
A Reverse AODV Routing Protocol in Ad Hoc Mobile Networks
}

\author{
Chonggun Kim, Elmurod Talipov, and Byoungchul Ahn \\ Department of Computer Engineering, \\ Yeungnam University, Korea \\ cgkim@yu.ac.kr, elmurod@ynu.ac.kr, b.ahn@yu.ac.kr
}

\begin{abstract}
In mobile ad hoc networks, mobile devices wander autonomously for the use of wireless links and dynamically varying network topology. AODV (Ad-hoc on-demand Distance vector routing) is a representative among the most widely studied on-demand ad hoc routing protocols. Previous protocols have shown some shortcomings on performance. AODV and most of the ondemand ad hoc routing protocols use single route reply along reverse path. Rapid change of topology causes that the route reply could not arrive to the source node, i.e. after a source node sends several route request messages, the node obtains a reply message, especially on high speed mobility. This increases both in communication delay and power consumption as well as decrease in packet delivery ratio. To avoid these problems, we propose a reverse AODV which tries multiple route replies. The extended AODV is called reverse AODV (R-AODV), which has a novel aspect compared to other on-demand routing protocols on Ad-hoc Networks: it reduces path fail correction messages and obtains better performance than the AODV and other protocols have. We design the R-AODV protocol and implement simulation models using NS-2. Simulation results show that the reverse AODV provides good experimental results on packet delivery ratio, power consumption and communication delay.
\end{abstract}

Keywords: AODV, Reverse AODV, NS-2, Simulation, Performance, Packet delivery ratio, communication delay.

\section{Introduction}

A mobile ad hoc network is a dynamically self-organizing network without any central administrator or infrastructure support. If two nodes are not within the transmission range of each other, other nodes are needed to serve as intermediate routers for the communication between the two nodes [1]. Moreover, mobile devices wander autonomously and communicate via dynamically changing network. Thus, frequent change of network topology is a tough challenge for many important issues, such as routing protocol robustness, and performance degradation resiliency [2-12].

Proactive routing protocols require nodes to exchange routing information periodically and compute routes continuously between any nodes in the network, regardless of using the routes or not. This means a lot of network resources such as energy and bandwidth may be wasted, which is not desirable in MANETs where the resources are constrained [1-3]. On the other hand, on-demand routing protocols don't exchange 
routing information periodically. Instead, they discover a route only when it is needed for the communication between two nodes [1, 6, 7]. Due to dynamic change of network on ad hoc networks, links between nodes are not permanent. In occasions, a node can not send packets to the intended next hop node and as a result packets may be lost. Loss of packets may affect on route performance in different ways. Among these packet losses, loss of route reply brings much more problems, because source node needs to re-initiate route discovery procedure.

A drawback of existing on-demand routing protocols is that their main route discovery mechanisms are not well concerned about a route reply message loss. More specifically, most of today's on-demand routing is based on single route reply message. The lost of route reply message may cause a significant waste of performance.

In this study we propose reverse AODV which has a novel aspect compared to other on-demand routing protocols on ad-hoc networks. In R-AODV, route reply message is not unicast, rather, destination node uses reverse RREQ to find source node. It reduces path fail correction messages and can improve the robustness of performance. Therefore, success rate of route discovery may be increased even though high node mobility situation. The simulation results show our proposed algorithm improves performance of AODV in most metrics, including packet delivery ratio, average end to end delay and power consumption.

\section{Motivation}

In mobile ad hoc networks nodes may move from one location to another on variety of node speed. As the result, the network topology changes continuously and unpredictably. Only within a short period of time neighboring nodes can loose communication link, especially when the mobility is high. In on-demand routing protocols, loosing a communication link between nodes brings route breaks and packet losses. Especially, loosing the RREP of AODV protocol produces a large impairment on the AODV protocol.

In fact, a RREP message of AODV is obtained by the cost of flooding the entire network or a partial area [1-5]. RREP loss leads to source node reinitiate route discovery process which causes degrade of the routing performance, like high power consumption, long end-to-end delay and inevitably low packet delivery ratio. Therefore, we are considering how simply to decrease the loss of RREP messages.

We can see a situation in Figure 1, where $S$ is a source node, D is a destination node and others are intermediate nodes. In traditional AODV, when RREQ is broadcasted by node $S$ and each node on a path builds reverse path to the previous node, finally the reverse path $\mathrm{D} \rightarrow 3 \rightarrow 2 \rightarrow 1 \rightarrow \mathrm{S}$ is built. This reverse path is used to deliver RREP message to the source node $\mathrm{S}$. If node 1 moves towards the arrow direction and goes out of transmission range of node 2, RREP missing will occur and the route discovery process will be useless. We can easily know that several alternative paths built by the RREQ message are ignored.

There are some possibilities that after sending a number of RREQ messages, source node can obtain a route reply message. As mentioned in [3], when the number of nodes is 100 and the number of flows is 50, 14\% of total RREP messages are lost. 


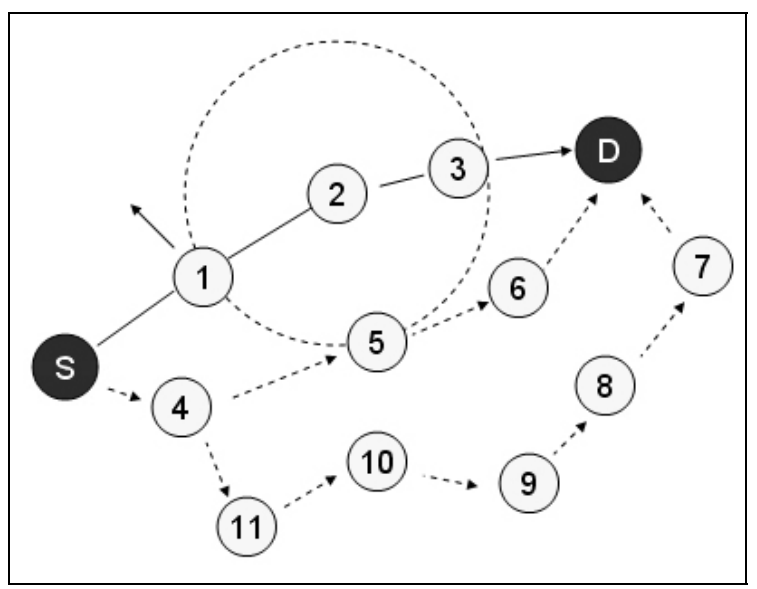

Fig. 1. RREP Delivery Fail

We propose the R-AODV to avoid RREP loss and improve the performance of routing in MANET. R-AODV uses absolutely same procedure of RREQ of AODV to deliver route reply message to source node. We call the route reply messages reverse request $(R-R R E Q)$. R-AODV protocol can reply from destination to source if there is at least one path to source node. In this manner, R-AODV prevents a large number of retransmissions of route request messages, and hence diminishes the congestion in the network. Moreover, R-AODV will improve the routing performance such as packet delivery ratio and end-to-end delay.

\section{Proposed R-AODV Protocol}

In this section we present an overview and purpose of proposed R-AODV protocol.

\subsection{Protocol Overview}

Analyzing previous protocols, we can say that most of on-demand routing protocols, except multipath routing, uses single route reply along the first reverse path to establish routing path. As we mentioned before, in high mobility, pre-decided reverse path can be disconnected and route reply message from destination to source can be missed. In this case, source node needs to retransmit route request message. Purpose of our study is to increase possibility of establishing routing path with less RREQ messages than other protocols have on topology change by nodes mobility.

Specifically, the proposed R-AODV protocol discovers routes on-demand using a reverse route discovery procedure. During route discovery procedure source node and destination node plays same role from the point of sending control messages. Thus, after receiving RREQ message, destination node floods reverse request (R-RREQ), to find source node. When source node receives an R-RREQ message, data packet transmission is started immediately. 


\subsection{Route Discovery in R-AODV}

Since R-AODV is reactive routing protocol, no permanent routes are stored in nodes. The source node initiates route discovery procedure by broadcasting. The RREQ message contains following information (Figure 2): message type, source address, destination address, broadcast ID, hop count, source sequence number, destination sequence number, request time (timestamp).

\begin{tabular}{||c|c||}
\hline Type & Reserved \\
\hline Broadcast ID \\
\hline Destination IP address \\
\hline Destination Sequence Number \\
\hline Source IP address \\
\hline Source Sequence number \\
\hline Request Time \\
\hline
\end{tabular}

Fig. 2. RREQ Message Format

Whenever the source node issues a new RREQ, the broadcast ID is incremented by one. Thus, the source and destination addresses, together with the broadcast ID, uniquely identify this RREQ packet $[1,9]$. The source node broadcasts the RREQ to all nodes within its transmission range. These neighboring nodes will then pass on the RREQ to other nodes in the same manner. As the RREQ is broadcasted in the whole network, some nodes may receive several copies of the same RREQ. When an intermediate node receives a RREQ, the node checks if already received a RREQ with the same broadcast id and source address. The node cashes broadcast id and source address for first time and drops redundant RREQ messages. The procedure is the same with the RREQ of AODV.

When the destination node receives first route request message, it generates so called reverse request (R-RREQ) message and broadcasts it to neighbor nodes within transmission range like the RREQ of source node does.

R-RREQ message (Figure 3) contains following information: reply source id, reply destination id, reply broadcast id, hop count, destination sequence number, reply time (timestamp).

When broadcasted R-RREQ message arrives to intermediate node, it will check for redundancy. If it already received the same message, the message is dropped, otherwise forwards to next nodes.

\begin{tabular}{|c|c||}
\hline Type & Reserved \\
\hline Broadcast ID \\
\hline Destination IP address \\
\hline Destination Sequence Number \\
Source IP address \\
\hline Reply Time \\
\hline
\end{tabular}

Fig. 3. R-RREQ Message Format 
Furthermore, node stores or updates following information of routing table:

$\square$ Destination Node Address

$\square \quad$ Source Node Address

$\square \quad$ Hops up to destination

$\square \quad$ Destination Sequence Number

$\square \quad$ Route expiration time and next hop to destination node.

And whenever the original source node receives first R-RREQ message it starts packet transmission, and late arrived R-RREQs are saved for future use. The alternative paths can be used when the primary path fails communications.

Let's see the same case of AODV, we have mentioned above, in figure 4. In RAODV, destination does not unicast reply along pre-decided shortest reverse path $\mathrm{D} \rightarrow 3 \rightarrow 2 \rightarrow 1 \rightarrow \mathrm{S}$. Rather, it floods R-RREQ to find source node S. And forwarding path to destination is built through this R-RREQ. Following paths might be built: $S \rightarrow 4 \rightarrow 5 \rightarrow 6 \rightarrow D, S \rightarrow 11 \rightarrow 10 \rightarrow 9 \rightarrow 8 \rightarrow 7 \rightarrow D$, and etc. Node $S$ can choose best one of these paths and start forwarding data packet. So RREP delivery fail problem on AODV does not occur in this case, even though node 1 moves from transmission range.

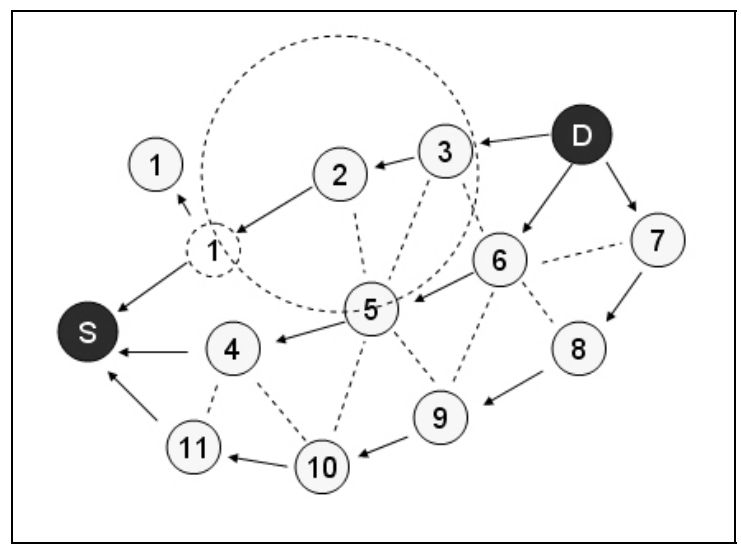

Fig. 4. R-RREQ From Destination to Source Node

\subsection{Route Update and Maintenance}

When control packets are received, the source node chooses the best path to update, i.e. first the node compares sequence numbers, and higher sequence numbers mean recent routes. If sequence numbers are same, then compares number of hops up to destination, routing path with fewer hops is selected. Since the wireless channel quality is time varying, the best path varies over time.

The feedback from the MAC layer can be used to detect the connectivity of the link. When a node notifies that its downstream node is out of its transmission range, the node generates a route error (RERR) to its upstream node. If fail occurs closer to destination node, RRER received nodes can try local-repair, otherwise the nodes 
forward RRER until it reaches the source node [1,2]. The source node can select alternative route or trigger a new route discovery procedure.

\subsection{Control Packet Overhead}

Intuitively, we can say that R-AODV causes a lot of control packet overhead. However, we can prove that route discovery procedure based on single reply message may cause even more packet overhead for some cases. We define the followings:

An ad hoc network has $\mathrm{N}$ number of nodes

Required number of control messages to discover routing path for AODV is $\operatorname{AODV}(N)$

Required number of control messages to discover routing path for R-AODV is $R A O D V(N)$.

Let's say m nodes participate to discover a routing path. Then AODV obtains a routing path using control message shown in (1), if it does not fail in first try.

$$
A O D V(m)=(m-1+t),
$$

where $t$ is the number of nodes relied on route reply message.

If source node fails in first try, because route reply message could not arrive, the node re-initiates path discovery, the number of control messages increase by the number of tries expressed in function (2).

$$
A O D V(m)=c(m-1+t),
$$

where $c$ is the number of tries for route discovery.

When we assume that R-AODV has at least one stable path by a REQ, then the number of control messages for R-AODV is in function (3). It will require only $2 \mathrm{~m}-2$ messages for route discovery.

$$
R A O D V(m)=\mathrm{O}(2 m-2) .
$$

So we can conclude when $c>1$, then AODV causes more packet overhead than the case of $c=1$ on $\mathrm{R}-\mathrm{AODV}$ routing.

\section{Performance Results}

In this section, we first describe the simulation environment used in our study and then discuss the results in detail.

\subsection{Simulation Environment}

Our simulations are implemented in Network Simulator (NS-2) [13] from Lawrence Berkeley National Laboratory (LBNL). The simulation parameters are as follows:

Number of nodes: $10,20,30,40,50$, respectively;

Testing area: $1000 \mathrm{~m} \times 1000 \mathrm{~m}$; 
Mobile speed: uniformly distributed between 0 and MAXSPEED (we choose MAXSPEED = 2, 5, 10, 25, 50,75m/s, respectively);

$\square$ Mobility model: random way point model (when the node reaches its destination, it pauses for several seconds, e.g., 1s, then randomly chooses another destination point within the field, with a randomly selected constant velocity);

$\square \quad$ Traffic load: UDP, CBR traffic generator;

$\square \quad$ Radio transmission range: $250 \mathrm{~m}$; and

$\square \quad$ MAC layer: IEEE 802.11.

Each simulation is run for 100 seconds and repeated for 10 times. We compared our proposed R-AODV with AODV.

\subsection{Results}

To evaluate performance of R-AODV with that of AODV protocol, we compare them using four metrics:

Delivery Rate: the ratio of packets reaching the destination node to the total packets generated at the source node.

$\square \quad$ Average End-to-End Delay: the interval time between sending by the source node and receiving by the destination node, which includes the processing time and queuing time.

Average Energy Remained: mean value of energy remained in each node.

$\square$ Control Overhead: sum of all route request messages, route reply messages and route error messages.

First, we can see performance according to increasing number of nodes. Figure 5 shows packet deliver ratio of AODV and R-AODV, by increasing number of nodes brings apparent difference between the two protocols, more exact result is shown on Figure 6.

Packet delivery ratio difference in figure 6 calculated as below

$$
\text { Difference }=\frac{\text { Delivery Ratio of } R-A O D V-\text { Delivery Ratio of } A O D V}{\text { Delivery Ratio of AODV }} \times 100 \%
$$

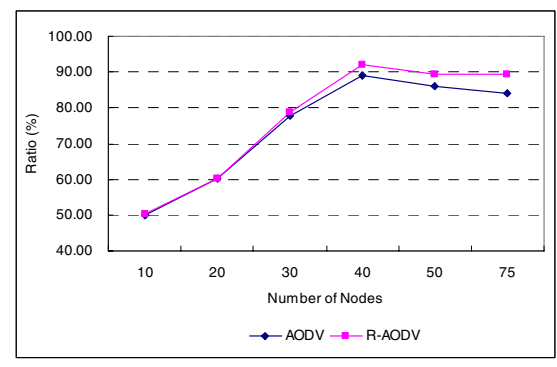

Fig. 5. Packet Delivery Ratio, when the number of nodes varies

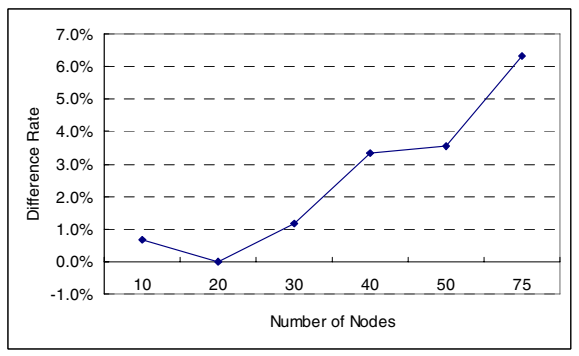

Fig. 6. Packet delivery ratio difference between two protocols, when the number of nodes varies 
Figure 7 shows the average end-to-end delay of each protocol. It should be noted that the delay is considered for the packets that actually arrive at the destinations. We can see that R-AODV has lower delay than AODV. The reason is that AODV chooses route earlier, R-AODV chooses recent route according to reverse request.

Figure 8 shows the average energy remained of each protocol. We have to mention that it is a mean value of energy remained each node at the end of simulation. Remained energy in R-AODV is higher than AODV; even it has sent more data packets to destination as shown on figure 5 and 6.

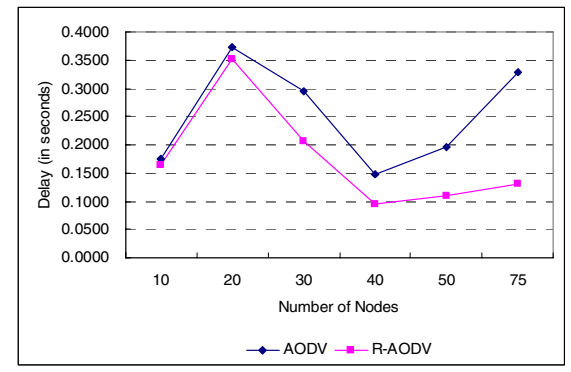

Fig. 7. Average end to end delay, when the number of nodes varies

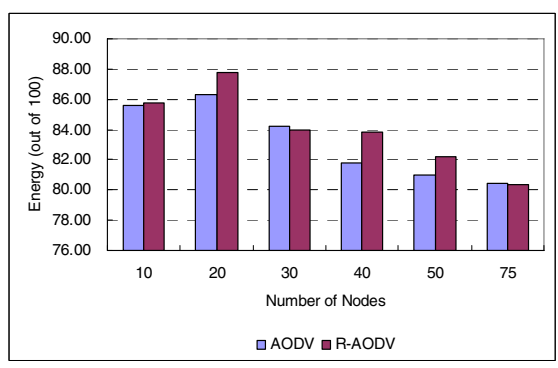

Fig. 8. Average energy remained, when the number of nodes varies

Figure 9 shows the control packet overhead required by the transportation of the routing packets. AODV has less control packet overhead. The reason is that R-AODV floods route reply message, but route reply message in AODV is unicast along reverse path. So we can say that, half of these messages are R-RREQ.

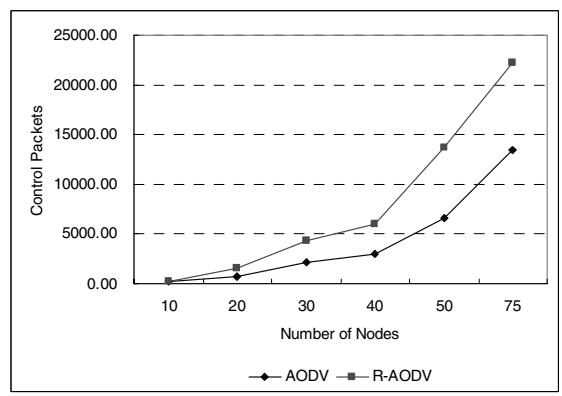

Fig. 9. Control Packet Overhead, when the number of nodes varies

Figure 10 shows packet delivery ratio of each protocols on varying node speed. In all cases, R-AODV shows better performance in packet delivery ratio.

Figure 11 shows average end to end delay where maximum speed of node varies. As fast node mobility causes high topology changes, recently selected path may have better consistency. 


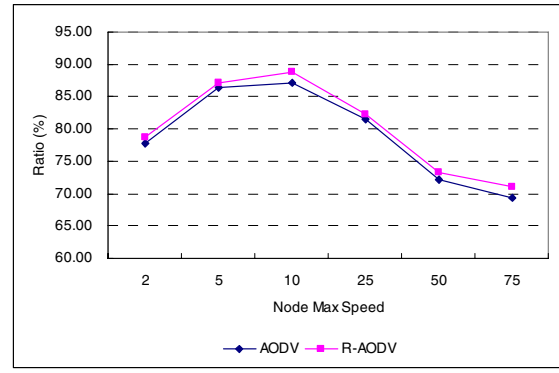

Fig. 10. Packet Delivery Ratio, when node speed varies

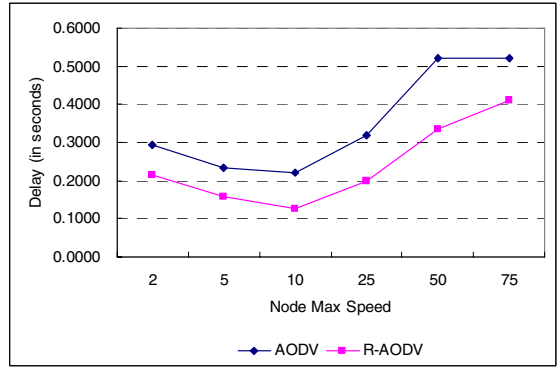

Fig. 11. Average end to end delay, when node speed varies

Figure 12 shows remained average energy. Where R-AODV has more remained energy than AODV, which will be helpful for nodes to survive in network.

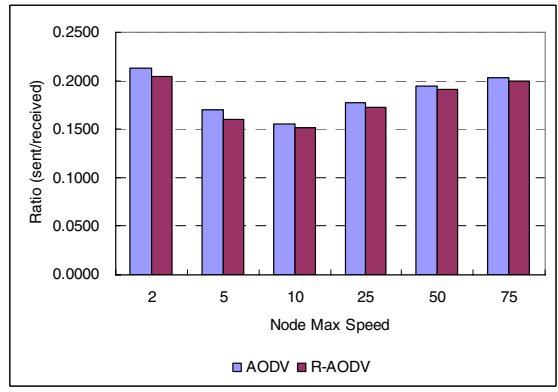

Fig. 12. Average remained energy, when node speed varies

\section{Conclusions}

Successful delivery of RREP messages are important in on-demand routing protocols for ad hoc networks. The loss of RREPs causes serious impairment on the routing performance. This is because the cost of a RREP is very high. If the RREP is lost, a large amount of route discovery effort will be wasted. Furthermore, the source node has to initiate another round of route discovery to establish a route to the destination. We proposed the idea of reverse AODV, which attempts reverse RREQ. R-AODV route discovery succeeds in fewer tries than AODV. We conducted extensive simulation study to evaluate the performance of RAODV and compared it with that of AODV using NS-2. The results show that R-AODV improves the performance of AODV in most metrics, as the packet delivery ratio, end to end delay, and energy consumption. Our future work will focus on studying practical design and implementation of the R-AODV. Multipath routing is another topic we are interested in. 


\section{References}

1. C. E. Perkins and E. M. Royer, "Ad hoc on-demand distance vector routing," in Proc. WMCSA, New Orleans, LA, Feb. 1999, pp. 90-100.

2. Zhi Li and Yu-Kwong Kwok, "A New Multipath Routing Approach to Enhancing TCP Security in Ad Hoc Wireless Networks" in Proc. ICPPW 2005.

3. Rendong Bai and Mukesh Singhal, "Salvaging Route Reply for On-Demand Routing Protocols in Mobile Ad-Hoc Networks" in MSWIM 205, Montreal, Quebec, Canada. Oct 2005

4. C. K.-L. Lee, X.-H. Lin, and Y.-K. Kwok, "A Multipath Ad Hoc Routing Approach to Combat Wireless Link Insecurity”. Proc. ICC 2003, vol. 1, pp. 448-452, May 2003.

5. S.-J. Lee and M. Gerla, "Split Multipath Routing with Maximally Disjoint Paths in Ad Hoc Networks," Proc. ICC 2001, vol. 10, pp. 3201-3205, June 2001.

6. M. K. Marina and S. R. Das "On-Demand Multi Path Distance Vector Routing in Ad Hoc Networks," Proc. ICNP 2001, pp. 14- 23, Nov. 2001.

7. A. Nasipuri and S. R. Das, "On-Demand Multipath Routing for Mobile Ad Hoc Networks," Proc. ICCN 1999, pp. 64-70, Oct. 1999.

8. C. Perkins, E. Belding-Royer "Ad hoc on-Demand Distance Vector (AODV) Routing", RFC 3561, July 2003

9. I. Stojmenovic, M. Seddigh, J. Zunic, ”Dominating sets and neighbor elimination-based broadcasting algorithms in wireless networks", IEEE Transactions on Parallel and Distributed Systems, 2002, pp. 14-25.

10. J.Wu, and H. Li, "On Calculating Power-Aware Connected Dominating Sets for Efficient Routing in Ad HocWireless Networks", in Proc. of the 3rd Int'l Workshop on Discrete Algorithm and Methods for Mobile Computing and Commun., 1999, pp. 7-14.

11. Jae-Ho Bae, Dong-Min Kim, Tae-Hyoun Kim, Jaiyong Lee. An AODV-based Efficient Route Re-Acquisition Scheme in Ad Hoc Networks.

12. Y.Kim, J.Jung, S.Lee and C.Kim, “A Belt-Zone Method for Decreasing Control Messages in Ad Hoc Netowkrs” ICCSA 2006, LNCS 3982, pp 64-72, 2006.

13. NS, The UCB/LBNL/VINT Network Simulator (NS), http://www.isi.edu/nsnam/ns/, 2004. 